\title{
Ética y calidad en las investigaciones sociales en salud. Los desajustes de la realidad
}

\author{
Susana Ramírez Hita ${ }^{1}$
}

Resumen: Este artículo analiza los lineamientos éticos y la rigurosidad metodológica en las investigaciones que sirven de base para las intervenciones en salud. Se apoya en las investigaciones socioantropológicas llevadas a cabo por la autora en territorio boliviano en los últimos seis ańos para diversos organismos de cooperación. De los resultados obtenidos, nace la preocupación sobre las deficiencias teórico-metodológicas de las consultorías sobre las que se basan las intervenciones en el campo de la salud. A través de una perspectiva de antropología médica se analizan tres ejemplos: a) introducción de la terapia de hidratación oral; b) incorporación de la salud intercultural para mejorar la calidad de atención, y c) calidad de atención de personas viviendo con VIH/SIDA. Se muestra la necesidad de respetar los tiempos necesarios para realizar correctamente las investigaciones sociales, con el fin de implementar intervenciones eficaces, acordes con los aspectos socioculturales de las diversas poblaciones que conforman el país.

Palabras clave: salud, ciencias sociales, ética, cooperación internacional, Bolivia

\section{Ethics and quality in social research in health. Mismatches of reality}

\begin{abstract}
This article analyzes the ethical outlines and methodological rigor in research basis of health interventions. It is supported by socio anthropological research carry out by the author in Bolivian territory in the last six years for diverse cooperation organisms. The results obtained rise worries on theoreticalmethodological deficiencies about the consulting in which health interventions are based. Three examples are analyzed by a medical anthropology perspective: a) introduction of oral hydration therapy; b) incorporation of intercultural health to enhance health care quality; and c) health care quality of persons living HIV/AIDS. The need to respect proper timing to carry out accurately social studies is shown, with the aim to implement effective interventions, in agreement with socio cultural aspects of the diverse populations in the country.
\end{abstract}

Key words: health, social sciences, ethics, international cooperation, Bolivia

\section{Ética e qualidade na pesquisa social em saúde. Inadequaçóes da realidade}

Resumo: Este artigo analisa as linhas éticas e o rigor metodológico nas investigaçóes que servem de base para as intervençôes em saúde. Se apoia nas investigaçóes sócio-antropológicas levadas a cabo pela autora em território boliviano nos últimos seis anos para diversos organismos de cooperação. Dos resultados obtidos, nasce a preocupação sobre as deficiências teórico-metodológicas das consultorias sobre as quais se baseiam as intervençôes no campo da saúde. Através de uma perspectiva de antropologia médica se analisam três exemplos: a) introdução da terapia de hidrataçáo oral; b) incorporaçáo da saúde intercultural para melhorar a qualidade da atenção, e c) qualidade de atenção de pessoas vivendo com HIV/AIDS. Mostra-se a necessidade de respeitar os tempos necessários para realizar corretamente as investigaçôes sociais com a finalidade de implementar intervençôes eficazes, de acordo com os aspectos sócio-culturais das diversas populaçóes que conformam o país.

Palavras-chave: saúde, ciências sociais, ética, cooperação internacional, Bolivia

\footnotetext{
${ }^{1}$ Profesora de Antropología, Departamento de Antropología, Facultad de Ciencias Sociales y Jurídicas, Universidad de Tarapacá, Arica, Chile

Correspondencia: susanahita@yahoo.es
} 


\section{Las ciencias sociales y la responsabilidad ética en las intervenciones en salud}

Las intervenciones del Estado boliviano en el campo de la salud deben ser analizadas en el contexto más general de colaboración con cooperaciones internacionales, agencias de desarrollo y organizaciones no gubernamentales (ONGs), que intervienen tanto técnica como financieramente en el diseño de programas y políticas sanitarias. Estas intervenciones son la base de planificaciones y políticas que se dirigen a mejorar la salud de la población y suelen ser realizadas a través de consultorías.

A lo largo de los últimos seis años ${ }^{2}$ hemos leído y analizado numerosos informes como resultado de esas consultorías, detectando profundas limitaciones teóricas y evidenciando falencias metodológicas en estas "investigaciones". La mayoría de las consultorías son abordadas a partir de los Procedimientos de Asesoría Rápida (RAP, por Rapid Assessment Procedures). Esta estrategia, que en ocasiones se plantea como metodología para abordar la realidad, fue creada por científicos sociales que trabajaban en el campo de la cooperación, ante la necesidad de establecer una forma de recolección y análisis de los datos acorde con los tiempos institucionales de los proyectos de desarrollo. Los RAP se desarrollaron dentro de un contexto de investigación basado en criterios de mercado: máxima producción al mínimo costo. "Están destinados a clínicos sin formación en ciencias sociales y sin un entrenamiento específico en investigación social o cultural cualitativa de campo" $(1,2)$. Los autores enfatizan que, en la actualidad, hay unos 50 tipos de $\mathrm{RAP}(3)^{3}$, pero este procedimiento no asegura la calidad de los datos, dado que obliga a utilizar matrices rígidas. Si a ello se suma el escaso entrenamiento de los investigadores de campo, se comprende que no se garantice que el dato esté bien recogido. Sin embargo, son los instrumentos más utilizados por las cooperaciones, ONGs y agencias de desarrollo con el fin de realizar diagnósticos para conocer el estado de la situación previo a la intervención(4:39). Analizaremos varios ejemplos de consecuencias de los

\footnotetext{
${ }^{2}$ La autora residió en Bolivia entre 2004 y 2010.

3 Por ejemplo, Rapid anthropological procedures: qualitative methodologies for planning and evaluation of health-related programs.
}

RAP e investigaciones desde ciencias de la salud y sociales con poca rigurosidad metodológica y exclusión de reflexión teórica suficiente, y cómo ello repercute en intervenciones de programas y políticas poco efectivas que afectan, sin duda, la salud de la población, asunto que concierne a la ética en la intervención.

Una de las características de las ciencias sociales, y en concreto de la antropología, es la necesidad de "tiempo" suficiente para dar cuenta de la realidad que se pretende investigar. Es la condición por excelencia para observar las prácticas de los sujetos sociales objeto de estudio, imprescindibles para las intervenciones eficaces en salud. Entre lo que un sujeto dice y hace suele haber contradicciones; de ahí la necesidad de observación de las prácticas, aspecto que no puede ser abordado en períodos cortos 4

Como afirma Zemelman(5): "Al no tener conciencia de que se está dando un desajuste entre la teoría y la realidad que se pretende denotar, resulta que terminamos inventando realidades". De tal modo, es un error considerar que las investigaciones pueden desarrollarse en períodos cortos y sin incorporar además investigadores de visión integral y crítica. O pensar que cabe conocer "actitudes, comportamientos (o conocimientos) y prácticas" sobre cualquier problemática de salud a partir del uso de las técnicas de grupo focal, encuestas o entrevistas exclusivamente.

Si las técnicas son manejadas sin una claridad teórica en torno a su significado real, "terminamos por creer que la realidad que es posible estudiar es tan sólo aquella que la técnica permite analizar" (5:68). Estamos ante investigaciones que no cuestionan la realidad empírica, que se han visto reducidas a la mera apariencia de la recolección del dato, sin problematizar la temática que se investiga en su totalidad. Este tipo de investigaciones son las más comunes en las consultorías realizadas para obtener diagnósticos de salud como base y justificativo de la futura intervención.

A través de nuestro trabajo en Bolivia hemos cons-

\footnotetext{
${ }_{4}^{4}$ Por "períodos cortos" entendemos desde consultorías que no sobrepasan los 15 días o un mes hasta consultorías de un año que pretenden abordar todo el país o parte de él.
} 
tatado que numerosas consultorías ${ }^{5}$ son realizadas por profesionales que diagnostican o evalúan buscando justificar la intervención ideológicopolítica de las diversas agencias de desarrollo, las cuales se caracterizan por la homogeneización de programas y políticas, sin considerar, en muchos casos, los contextos locales. Es decir, los resultados suelen justificar la intervención de los diversos organismos y siempre en términos positivos. Cuando la investigación es realizada críticamente y con el tiempo necesario para llevarla a cabo, los resultados difieren de los informes presentados en las consultorías. Sin embargo, son estos últimos los que sirven de base para crear los programas de intervención y, en el caso en concreto de Bolivia, también políticas sanitarias.

Los resultados de estas investigaciones no han ayudado a entender, en toda su complejidad y magnitud, la incidencia real de la enfermedad y la atención en el país. Se trata de estudios con diversos sesgos, en especial en lo referente a la unidad de análisis seleccionada, la metodología empleada, las categorías aplicadas para la recolección de datos y el análisis de la información. Son estudios que pueden ser cuestionados desde el rigor metodológico, imprescindible en una buena investigación en el campo de las ciencias sociales.

En lo que concierne a la ética de investigación, entran en juego no sólo los investigadores (el tipo de investigación que se realiza, para quién y cuáles son los fines), también los organismos e instituciones que financian y contratan a los consultores o investigadores. Es común que no se contrate a consultores o investigadores críticos y, de hacerlo, en ocasiones se les persuade de modificar aquellas partes que no se encuentran dentro de los lineamientos de la institución. Esto lleva a casi un consenso, entre las personas cuya fuente laboral son las consultorías, de aceptar las condiciones que van implícitas y a veces explícitas en los contratos laborales.

Las contradicciones que aún hoy atraviesan las ciencias sociales se ponen de manifiesto en el contexto de la cooperación, en la aceptación de las investigaciones rápidas, ya que cada vez más

${ }^{5}$ Hemos revisado más de 100 informes de consultorías. el financiamiento proviene de organizaciones de cooperación o instancias que exigen acortar los plazos para la presentación de los resultados finales, incluso de las investigaciones que se llevan a cabo dentro de las universidades.

\section{Investigaciones en ciencias sociales y de la sa- lud: los desajustes con la realidad}

En este apartado incluiremos algunos ejemplos para argumentar que la ética no puede separarse de las investigaciones en ciencias sociales y de la salud, que todo estudio debería incorporar una mirada crítica que involucre el análisis de los aspectos macro y microsociales, y ello sólo puede realizarse adecuadamente a través de investigaciones que no se restrinjan a los tiempos institucionales de las agencias de desarrollo. Estas instituciones deberían preguntarse: ¿cuántos de sus proyectos han sido realmente exitosos? La mayoría de los informes e investigaciones revisados dan como resultado el éxito indiscutible de la intervención. Sin embargo, a través de nuestro trabajo de campo, tanto en el Altiplano como en la región del Oriente boliviano, hemos constatado que diversos libros, informes y estudios realizados en el campo de la salud no corresponden con la realidad o plantean un escenario sesgado. De tal manera, otorgan una imagen distorsionada de los reales problemas de salud.

A través de tres ejemplos demostraremos el argumento de las limitaciones teórico-metodológicas de las investigaciones realizadas en períodos cortos: a) la terapia del suero de rehidratación oral como estrategia para disminuir la diarrea y desnutrición y, con ello, la mortalidad infantil; b) la incorporación del concepto de salud intercultural en el altiplano para mejorar la calidad de atención en salud, y c) la calidad de atención a personas viviendo con VIH/SIDA en la región del Oriente del país. Veremos la importancia de incorporar los aspectos socioculturales y macrosociales para entender las deficiencias en la calidad de atención y en los programas que se implementan.

\section{La terapia de suero de rehidratación oral en el altiplano boliviano}

Una de las estrategias introducidas en los años 80 
en los centros de atención primaria del país fue la terapia del suero de rehidratación oral para disminuir la mortalidad infantil en los procesos de diarrea. Esta medida de hidratación oral comunitaria se llevó a cabo por diversos organismos internacionales ${ }^{6}$. Entregar gratuitamente los sueros en los centros de atención primaria presuponía que la población los consumía, de tal manera que quedaba registrada la entrega del suero y el éxito de la estrategia. Sin embargo, no ayudó a disminuir la mortalidad infantil y no fueron realizadas investigaciones o evaluaciones que determinaran cuál era el motivo por el cual la población indígena altiplánica no consumía el suero a pesar de recogerlo en los servicios de salud.

Cuando se realiza trabajo de campo en el interior de los hogares, observando las prácticas se ve que el suero de rehidratación oral no se administra ni a bebés ni a niños. El personal sanitario alega que el motivo es que tiene "feo sabor". Pero existen otras razones más importantes.

En las culturas aimara y quechua la concepción de salud/enfermedad está basada en la relación frío y calor. El cuerpo necesita el equilibrio entre lo frío y lo caliente para estar sin ninguna enfermedad. Las enfermedades pueden ser frías o cálidas y los remedios (sean medicamentos de patente o caseros) deben ser de temperamento contrario. Es decir, que si una enfermedad es considerada cálida el remedio que debe tomarse es frío y viceversa. De no poseer esa cualidad, la madre no entregará a su niño el remedio, ya que considera que "le hará mal” en vez de sanarlo. Algunas de las enfermedades pueden tener ambos temperamentos, dependiendo de cuál es el origen de la misma(6).

Según el origen de la enfermedad, hay al menos 11 tipos de diarreas diferentes. Algunas son provocadas por enfermedades espirituales, en cuyo caso sólo está capacitado para atenderlas el terapeuta tradicional, y otras son consideradas enfermedades corporales que, dependiendo del origen, puede tratarse de cálidas o frías(7). Si la diarrea es considerada cálida y el suero de rehidratación oral caliente, dentro de la concepción de salud local el remedio es contraproducente. El temperamento

\footnotetext{
${ }^{6}$ Uno de los organismos que más colaboró fue UNICEF.
}

cálido o frío de los remedios se relaciona, entre otros aspectos, con el sabor (picante, amargo, etc.) y la temperatura.

Esta terapia de suero de rehidratación oral, que actualmente se imparte a través de la estrategia de Atención Integrada a las Enfermedades Prevalentes de la Infancia (AIEPI), no se analizó desde el aspecto sociocultural y tampoco se reconoció que no cumplió con su objetivo. Para entender por qué no funcionaba hacía falta una investigación socioantropológica y, de esa manera, conocer cuáles eran los sueros caseros acordes con las concepciones del frío y el calor que son los que la población consume.

La mortalidad infantil causada por la diarrea y la desnutrición es mucho más compleja, y es necesario realizar investigaciones serias que articulen el trabajo de campo con la reflexión teórica para crear programas eficaces y coherentes con la realidad. Bolivia posee 36 poblaciones indígenas diferentes, con diversas formas de entender la salud y la enfermedad, a las que, de no ser conocidas, es difícil que los programas de atención primaria respondan eficazmente.

Los aspectos socioculturales son esenciales para conocer las formas de entender y atender la salud y la enfermedad de los distintos grupos sociales, pero al mismo tiempo no pueden dejarse de lado los aspectos macrosociales para entender por qué diversos programas se trasladan del exterior a ámbitos locales sin contextualizar. De aquí la importancia de las investigaciones socioantropológicas de larga duración y del análisis crítico, previos al diseño de programas y políticas de salud.

\section{Introducción del concepto de salud intercultu- ral en la salud pública boliviana}

En el caso boliviano, el concepto de salud intercultural fue introducido por la cooperación internacional. Los planificadores asumieron que si la población no recurría a los servicios de salud era debido a que la mayoría indígena se atendía con su medicina tradicional. Como estrategia para disminuir la mortalidad en las mujeres y los niños se decidió incentivar financiera y técnicamente lo 
que se llamó "salud intercultural"”. Esto consistía en reforzar los centros de atención primaria con los terapeutas tradicionales y parteras para que las mujeres no tuvieran temor de realizar el parto en los servicios de salud. Al mismo tiempo, se capacitaba al personal sanitario sobre aspectos de la medicina tradicional andina y su forma de entender y atender la salud. De esa manera, se pretendía mejorar la calidad de atención de los servicios de salud, sobre los que había numerosas quejas por parte de la población.

Se realizaron diversos estudios e investigaciones, en su mayoría por personal sanitario que hizo el postgrado en Interculturalidad y Salud ${ }^{8}$, financiado y coordinado por la cooperación internacional, y que posteriormente asumió el Ministerio de Salud y Deportes. El posgrado otorgaba una mínima capacitación en metodología cualitativa, confundiendo la utilización de técnicas cualitativas (entrevistas, grupos focales, etc.) con las investigaciones socioantropológicas con metodología cualitativa. A partir de ese posgrado se generó toda una serie de estudios con el título de cualitativos que sirvieron de base, a pesar de sus limitaciones metodológicas, para seguir respaldando la propuesta inicial que sustentaba que la solución a los elevados índices de mortalidad materna e infantil era la incorporación de la salud intercultural.

En 2008 el concepto de salud intercultural pasó incluso a formar parte de la Nueva Constitución Política del Estado. En 2007, el Ministerio de Salud y Deportes de Bolivia llegó a definirlo de la siguiente manera: "La interculturalidad en la salud es el enfoque sociocultural del ejercicio de la práctica médica con personas culturalmente distintas, donde cada una de ellas se predispone al diálogo, respeto, reconocimiento, valoración y aceptación de los diferentes saberes médicos, promoviendo su articulación y complementariedad para mejorar la capacidad de actuar de manera equilibrada

\footnotetext{
${ }^{7}$ La salud intercultural comenzó a desarrollarse a fines de la década de los 90 y comienzos de 2000 por la cooperación internacional, y fue asumida por el Ministerio de Salud y Deportes en 2006 con la creación del Viceministerio de Medicina Tradicional e Interculturalidad.

${ }^{8}$ Del posgrado en Interculturalidad y Salud han salido hasta el momento cuatro promociones en el país, dos en La Paz y dos en Potosí.
}

en la solución de los problemas de salud: calidad de atención, conocimientos, creencias y prácticas de salud diferentes" (8). Esta definición se apoya en las consultorías e investigaciones sobre la salud intercultural realizadas en Bolivia y en la opinión de los egresados del mencionado posgrado.

En la definición se excluyen elementos importantes, como que el personal sanitario y el paciente pertenecen a la misma cultura en la zona andina (aimara o quechua), y aspectos macrosociales, como que la situación de pobreza y desigualdad social afecta no sólo a las poblaciones indígenas sino también al personal sanitario. La desocupación y las deficientes condiciones laborales quedan solapadas en el concepto de "cultura" (implícito en el término de interculturalidad), que oculta las situaciones de desigualdad y pobreza del propio personal sanitario, algo que también forma parte de la relación que se establece con el paciente(4)

El análisis sobre el problema de los deficientes índices epidemiológicos del país se centró en lo cultural y en el desentendimiento entre el personal sanitario y la población. Se partió de que el problema sanitario en Bolivia radicaba básicamente en los hábitos culturales y la no respuesta de la población de acudir a los servicios de salud, por ello había que transformar éstos a las costumbres indígenas.

Años más tarde de la implementación de la medida, realizamos una investigación sobre la calidad de atención en estos servicios ${ }^{10}$. Lo que encontramos fue que, a pesar de incorporar los aspectos culturales a los servicios de salud, la población seguía sin recurrir a ellos.

En el énfasis otorgado al reconocimiento de la medicina tradicional y su institucionalización se obvió el análisis de que en las zonas donde se crearon los centros de salud intercultural (el departamento de Potosí y la provincia Bautista Saavedra del departamento de La Paz) la esperanza de vida es una de las más bajas del país y que, a su vez, son áreas donde la población está atendida sobre todo mediante la medicina tradicional, por la falta de

\footnotetext{
${ }^{9}$ En este artículo no podemos extendernos más sobre el tema, pero recomendamos ver la investigación completa(4). ${ }^{10} \mathrm{La}$ investigación se realizó entre 2007 y 2008.
} 
una salud pública eficiente. Como estrategia política se incorporó la medicina tradicional dentro de los centros, pero no se trabajó en el fortalecimiento de los servicios de salud en cuanto a capacidad resolutiva. Fue una manera fácil y económica de hacer visibles los cambios en el ámbito de la salud. Esta estrategia, que se propuso como solución a ciertas falencias de la salud pública, no ayudó sin embargo a mejorar la calidad de los centros y hospitales - atendidos por estudiantes sin supervisión que remplazan a profesionales - ni las deficientes condiciones laborales(4).

Muchos de los trabajos que sustentaron teóricamente el concepto de la salud intercultural partieron de consultorías realizadas y publicadas que señalaban el éxito rotundo de la intervención y la mejora de la salud pública. El propio Ministerio de Salud y Deportes creó en los últimos años una línea editorial(9-11) en la que publicó documentos técnicos realizados a partir de investigaciones de consultorías rápidas. Estos estudios se caracterizan por estar centrados en la atención al parto $y$, a su vez, por el hecho de que los protocolos para incorporar cambios en la atención están únicamente dirigidos a las poblaciones aimara y quechua, a pesar de que Bolivia cuenta con 36 poblaciones indígenas diferentes, además de poblaciones mixtas, criollos, mestizos, etc. La calidad de la atención se centró en el desentendimiento cultural mientras que el énfasis se colocó en la necesidad de cursos de capacitación para mejorar la salud pública. Son investigaciones y documentos técnicos en los que el eje se situó de manera exclusiva en los aspectos culturales.

De la misma manera, encontramos estudios realizados por agencias de desarrollo, nacionales o internacionales, que han descrito sus experiencias en artículos o libros. Se caracterizan por exponer detalladamente el éxito del proyecto y enfatizan el aspecto participativo del mismo $(8,12-17)^{11}$. Muchos de estos proyectos, que aparecen como exitosos en artículos y libros, se han llevado a cabo en las mismas zonas en las cuales realizamos el trabajo de campo y donde evidenciamos que

\footnotetext{
${ }^{11}$ Existe una extensa bibliografía de material generado por ONGs y agencias de desarrollo, pero aquí nos centraremos exclusivamente en los autores que han influenciado teóricamente la implementación de los centros de salud intercultural en la zona andina.
}

el carácter de "exitosos" dista mucho de la realidad. Teóricamente, estos estudios se sustentaron en el trabajo que, para agencias de cooperación, realizan profesionales de las ciencias sociales con escasa reflexión teórica, entre otras razones porque las condiciones laborales no lo permiten. Esto lleva, en ocasiones, a no revisar estudios anteriores sobre el tema y a poner todo el énfasis de la salud intercultural exclusivamente en la cultura como manera de mejorar la salud del país, sin analizar ningún otro aspecto. La mayoría de estos trabajos están basados en las diferencias culturales y no en las desigualdades sociales.

Es importante señalar que las propuestas de cambio del Ministerio de Salud y Deportes se sustentan en las aportaciones de ciertos expertos de las ciencias sociales que carecen de reflexión crítica y que, por un lado, sólo reconocen dos sistemas de atención a la salud — biomédico y tradicionaly no la diversidad de formas de atención, y, por otro, avalan las investigaciones rápidas e incentivan que las mismas las realicen clínicos para, a su vez, efectuar las intervenciones en los programas de desarrollo. La antropología médica ya ha demostrado desde hace décadas la existencia del pluralismo en las formas de atención a la salud, pluralismo al que recurre la mayoría de los grupos sociales. Sin embargo, no parece que ello haya sido asumido por parte de los profesionales que teóricamente están incentivando la línea de intervención en salud intercultural.

\section{Calidad de atención en salud en personas vi- viendo con VIH/SIDA en la región del Oriente boliviano}

En 2009 realizamos una investigación sobre la calidad de atención de salud en personas viviendo con VIH/SIDA (PVVS) en la región del Oriente boliviano, a partir de los saberes de las PVVS, de sus cuidadores y del personal sanitario que los atiende, al mismo tiempo que se realizó observación en los centros departamentales de vigilancia y referencia (CDVIR) —espacios en lo que se atiende a trabajadoras sexuales y a personas viviendo con VIH/SIDA - así como en los hospitales públicos. 
Parte de la investigación consistió en la revisión bibliográfica de informes, libros, estudios, etc. en torno al VIH/SIDA en Bolivia(18-22), comprobando que sus resultados no habían ayudado a entender, en toda su complejidad y magnitud, la incidencia real de la enfermedad en el país. Expondremos algunos ejemplos de estos trabajos para ver las limitaciones teórico-metodológicas de las investigaciones rápidas sobre la actual condición de las personas viviendo con VIH/SIDA.

Vamos a examinar dos trabajos: uno realizado desde las ciencias sociales y otro desde las ciencias de la salud. El primero es una consultoría realizada por un equipo multidisciplinario y publicada posteriormente con el título "Pueblos indígenas, ITS, VIH y SIDA. Infecciones de transmisión sexual, VIH y SIDA en comunidades indígenas de Pando: una aproximación a conocimientos, actitudes y prácticas de poblaciones adultas y jóvenes". La finalidad del estudio era proponer recomendaciones de actuación entre las poblaciones Tacana, Cavineño, Ese-ejja, Yaminahua y Machineri(18). Los investigadores recomendaban considerar determinados factores socioculturales en el diseño e implementación de estrategias de prevención del VIH. Sin embargo, estos factores no aparecen en el informe.

El trabajo se llevó a cabo en cinco comunidades representativas de cada uno de los pueblos indígenas seleccionados, con hombres y mujeres de $14 \mathrm{a}$ 24 años de edad y de 25 a 45. Los investigadores consultaron a un total de 85 personas, utilizaron la técnica de grupo focal (cada grupo fue integrado entre cinco a 15 personas) y una enfermera y una investigadora social llevaron a cabo entrevistas. El período que diseñaron para la recolección de la información fue de tres semanas y, posteriormente, el material fue analizado por una antropóloga. La pretensión de esta investigación era conocer los conocimientos, actitudes y prácticas sobre ITS, VIH y SIDA de los grupos indígenas de la zona amazónica de Pando.

No obstante los investigadores reconocen las limitaciones del estudio - los grupos focales y "consultas" a varones fueron llevados acabo por las mujeres que recogieron la información en campo-, no permanecieron en las comunidades, sólo estuvieron presentes para realizar los grupos focales, momentos en los que reunieron a la población indígena de cualquier grupo étnico, como si por el solo hecho de ser indígenas tuvieran la misma concepción sobre la sexualidad y sobre salud/enfermedad/atención.

Esto visibiliza la confusión entre la utilización de técnicas cualitativas y lo que significa realizar una investigación con metodología cualitativa. Esta última no puede ser realizada en períodos cortos. Este estudio se hizo para ofrecer recomendaciones de acción, pero la base desde la cual se pensó la investigación fue mal planteada y las técnicas utilizadas fueron inadecuadas.

Un ejemplo de la incongruencia metodológica es que la información sobre ITS/VIH/SIDA a varones fue obtenida por mujeres. Un grupo focal realizado de una manera adecuada implica, en muchos casos, meses de preparación con quienes se va a trabajar, si se quiere realmente tener información que represente la realidad. Las prácticas sobre sexualidad, ITS/VIH/SIDA no pueden ser registradas adecuadamente mediante las técnicas de grupo focal o entrevista; para obtener prácticas de salud la técnica que sirve es la observación o la observación participante. Ambas técnicas de metodología cualitativa necesitan de un período largo de tiempo para ser llevadas a cabo correctamente. Ninguna investigación seria puede ser realizada en tres semanas de trabajo de campo y, menos aún, si se abarca cinco grupos étnicos.

Esta es sólo una muestra de la multitud de informes, estudios, investigaciones y libros que se concretan en recomendaciones y propuestas de intervención sobre la base de realidades poco contrastadas, de datos de campo cuestionables, pero que, lamentablemente, son la base de intervenciones y creación de programas de salud.

Otro de los ejemplos es una consultoría realizada por el Ministerio de Salud y Deportes(22). En esta ocasión, los investigadores pertenecen a las ciencias de la salud. El estudio se titula "Estudio sobre sexualidad masculina y VIH en Bolivia (SEMVBO)”. La investigación fue realizada en cua- 
tro ciudades (Santa Cruz, Cochabamba, La Paz y El Alto) y en cuatro meses. Aquí vuelve a confundirse lo que significa la utilización de técnicas con una investigación de metodología cualitativa. Los investigadores realizaron encuestas a un total de 1.019 hombres que tienen sexo con hombres. En el estudio confunden el término "encuesta" con el "término entrevista". Pretenden que en una encuesta, hecha durante 30 minutos, "cara a cara" y por personas que han sido adiestradas a lo largo de unas semanas(22), los sujetos sociales estén dispuestos a hablar de sus prácticas sexuales. Y más aún si los entrevistadores son "acompañante par" $^{\prime 2}$. Si uno de los fundamentos de mayor importancia en la investigación es acercarse el máximo posible a la objetividad, debe realizarse, consecuentemente, por agentes externos a la unidad de análisis (personal sanitario, personas viviendo con VIH/SIDA, familiares, etc.). Es un supuesto erróneo considerar que los actores inmersos en una problemática puedan conocer mejor dicha realidad y analizarla con objetividad. Por lo menos, no de forma global y en toda su complejidad; pueden conocer mejor aspectos parciales, aquellos que han experimentado y que, en su interrelación con el investigador, consiguen reflexionar y por tanto permitir a éste objetivar y situar su experiencia subjetiva en el contexto de la complejidad de los procesos sociales estudiados. Partimos de que la realidad es construida y necesita ser analizada; no existe en sí misma o, en cualquier caso, es parcial en tanto no sea problematizada. Los múltiples significados de la realidad no aparecen hasta que ésta se somete al cuestionamiento y la problematización. Y los actores internos no pueden, generalmente, mantener una mirada objetiva, alejada del problema concreto que les envuelve.

Si todo el esfuerzo se centra en cuantificar los enfermos de VIH/SIDA y aquellos que reciben tratamiento, se están dejando de lado los problemas reales de la epidemia; problemas que, sin duda, no son los de la mera cuantificación. El hecho de querer cuantificar todo aquello que se refiere inclusive a "comportamientos" y prácticas, supone abandonar la reflexión en torno a las falencias metodológicas, teóricas y epistémicas de las inves-

\footnotetext{
${ }^{12}$ Acompańante par, en este caso, se refiere a hombres que tienen sexo con hombres y VIH.
}

tigaciones que se efectúan a la hora de propiciar el diagnóstico, o con la voluntad de evidenciar la situación de la epidemia y la de quienes conviven con el virus.

Un ejemplo que puede servir para ilustrar este razonamiento sobre una base conceptual insuficiente se refiere a los términos "homosexual", "bisexual" y "heterosexual", los que, al igual que otros, no han sido definidos de acuerdo con el contexto específico boliviano. Tanto los investigadores como los organismos que impulsan campańas y programas de educación sanitaria en torno al VIH/SIDA (Ministerio de Salud, ONGs, agencias de cooperación) han dado por sentado que se trata de conceptos universales. No obstante, en el transcurso de nuestro trabajo de campo hemos apreciado las numerosas acepciones que, procedentes de los diversos grupos sociales y culturales, se otorgan a estos términos. En lo que concierne al VIH/SIDA consideramos que el método que puede llegar a un mayor acercamiento a la complejidad asociada a todos estos conceptos es el método etnográfico ${ }^{13}$.

Si se ignoran dichas concepciones referidas a la población, a sus grupos étnicos y poblaciones mixtas, no pueden encararse planificaciones posteriores eficaces. La eficacia estaría siempre supeditada al conocimiento en torno a las formas de entender y atender el VIH/SIDA por parte de los diversos grupos poblacionales. Es necesario, por tanto, conocer el significado que otorgan los distintos grupos sociales a la sexualidad, al riesgo y a determinados componentes que, de no ser conocidos, restan efectividad a la educación sanitaria que se pretende establecer para disminuir la enfermedad.

Por ello, consideramos que el primer paso para comenzar un cambio real es impulsar investigaciones serias, rigurosas, planteadas con el interés de incorporar todos los significados que influyen en la percepción social subjetiva y que se concretan en las prácticas propias de los centros de salud (públicos y privados), así como en los hogares y en la vida cotidiana de los distintos grupos sociales y culturales.

\footnotetext{
${ }^{13}$ Véase la contribución del método etnográfico al registro del dato epidemiológico(23).
} 
El énfasis que colocamos en las investigaciones es porque las consideramos importantes a la hora de crear estrategias para mejorar la calidad de atención. Nos referimos al concepto de "calidad" como "el óptimo entendimiento por parte de los actores involucrados en el acto médico. Un entendimiento que implica: tiempo adecuado para la consulta médica; que los servicios de salud cumplan con las mínimas condiciones propias de funcionamiento resolutivo, y que el personal sanitario esté en plenas condiciones para ejercer bien sus funciones, requisito éste último que conlleva un sueldo digno, acorde con el nivel profesional, el ejercicio pleno de los derechos laborales (vacaciones, aguinaldos, seguro de enfermedad, etc.), una buena formación académica $\mathrm{y}$, también, que los prestadores de servicios de salud otorguen un buen trato al paciente, el que debería incorporar el respeto a sus formas propias de entender y atender la salud y la enfermedad. Ello implicaría respetar los orígenes culturales, pero también aspectos como la religión, el género, la clase social y la identidad étnica, y que debe repercutir en una mejora de los índices epidemiológicos. Todos estos aspectos deberían ir acompañados de condiciones sociopolíticas que ayuden a su concreción. La calidad de atención no debe ser entendida exclusivamente como producto de responsabilidades individuales, sino también como obligaciones concernientes al propio Estado"(4:18).

Es necesario, por tanto, un replanteamiento de las investigaciones hechas a través de los RAP. Los organismos e instituciones que trabajan con progra- mas y proyectos de desarrollo deberían plantearse el cambio de los tiempos institucionales para las investigaciones previas a la intervención y para las evaluaciones de los proyectos que necesitan de investigaciones rigurosas, con el fin de comprobar si se han incorporado los cambios propuestos por el proyecto o programa. Este aspecto no puede ser llevado a cabo correctamente a través de la utilización de técnicas cualitativas, de las que se han apropiado los clínicos para justificar sus "investigaciones de metodología cualitativa”, las cuales llevan a intervenciones rápidas y a una ética de investigación cuestionable que influye directamente en la calidad de atención de los servicios de salud y en la salud de la población en general.

Es evidente la ausencia de control epistemológico que guíe las investigaciones, así como limitación en la capacidad crítica y una desarticulación entre el trabajo de campo y la reflexión teórica. En este tipo de investigaciones no hay una problemática clara a abordar, sino que el objetivo final es un proyecto a ejecutar que justifique la financiación obtenida. Hay, por tanto, un agotamiento del modelo vigente de intervención en lo que se refiere al abordaje de las problemáticas de salud, y ello se refleja en los escasos cambios de los índices de morbimortalidad que presenta Bolivia, a pesar de décadas de intervención en el campo de la salud.

\section{Agradecimientos}

A María Julia Castellón-Arrieta y a Oriol Romaní por sus valiosos aportes a este artículo. 


\section{Referencias}

1. Allué X, Mascarella L, Bernal M, et al. De la hegemonía de la clínica a la etnográfica en la investigación intercultural en salud. En: Fernández G, (eds.). Salud e interculturalidad en América Latina. Antropología de la salud y crítica intercultural. Ecuador: ABYA YALA, UCLM, AECI; 2006: 15-32.

2. Menéndez E. Técnicas cualitativas, problematización de la realidad y mercado de saberes. Cuadernos de Antropología Social, Facultad de Filosofía y Letras-UBA 2001; 13: 9-51

3. Scrimshaw S, Gleason G, (eds.) Rapid anthropological procedures: qualitative methodologies for planning and evaluation of health-related programs. Boston: International Nutrition Foundation for Developing Countries; 1992.

4. Ramírez Hita S. Calidad de atención en salud. Prácticas y representaciones sociales en las poblaciones quechuas y aimaras del altiplano boliviano. La Paz: OPS/OMS; 2009. Disponible en: http://www. ops.org.bo/textocompleto/nca30643.pdf

5. Zemelman H. Voluntad de conocer. El sujeto y su pensamiento en el paradigma crítico. México: Anthropos Editorial; 2005: 64.

6. Ramírez Hita S. Donde el viento llega cansado. Sistemas y prácticas de salud de la ciudad de Potosí. La Paz: Cooperación Italiana; 2005.

7. Pérez Z. Conocimientos y prácticas andinas en torno a las diarreas. El caso de Yura. Potosí: Postgrado de salud intercultural. Universidad Autónoma Tomás Frías; 2002.

8. Royder R, Angeloni M. Estándares e indicadores de calidad de atención con enfoque intercultural para centros de salud de primer nivel. La Paz: Cooperazione Italiana; 2007: 16.

9. Ministerio de Salud y Deportes. Guía para la atención intercultural de la salud materna. La Paz: Serie Documentos Técnicos Normativos; 2005.

10. Ministerio de Salud y Deportes. El enfoque intercultural en la atención de la salud materna: un avance para las politicas públicas de salud. La Paz: Documentos de investigación, publicación 2; 2007.

11. Ministerio de Salud y Deportes. Guía para facilitadores/as en atención intercultural de la salud materna. La Paz: Serie documentos de Técnico-Normativos, Publicación 14; 2007.

12. Dibbits I, De Boer M. Encuentros con la propia historia. Interculturalidad y trato humano desde las perspectivas de un equipo de salud. La Paz: Tahipamu; 2002.

13. Campos R. Experiencias sobre salud intercultural en América Latina. En Fernández G, (ed.) Salude interculturalidad en América Latina. Perspectivas antropológicas. Quito: ABYA YALA, BOLIHISPANA, UCLM; 2004: 129-151.

14. Campos R, Citarella L. Willaqkuna. Un programa de formación en salud intercultural en Bolivia. En Fernández G, (ed.) Salud e interculturalidad en América Latina. Perspectivas antropológicas. Quito: ABYA YALA, BOLIHISPANA, UCLM; 2004: 93-106.

15. Flores JA. Una etnografía del "año de provincias" y de "cuando no hay doctor". Perspectivas de salud intercultural en Bolivia desde la biomedicina convencional. En: Fernández G, (ed.) Salud e interculturalidad en América Latina. Perspectivas antropológicas. Quito: ABYA YALA, BOLIHISPANA, UCLM; 2004: 181-212.

16. Baixeras JL. Salud intercultural. Relación de la medicina kallawaya con el sistema de salud público en San Pedro de Curva. En: Fernández G, (ed.) Salud e interculturalidad en América Latina. Perspectivas antropológicas. Quito: ABYA YALA, BOLIHISPANA, UCLM; 2004: 271-287.

17. Fuertes A, Perez R. Encuentro de dos: la biomedicina y medicina tradicional en Tinguipaya. La Paz: Cooperazione Italiana y COOPI; 2007.

18. Family Care International/BOLIVIA. Pueblos indígenas, ITS, VIH y SIDA. Infecciones de transmisión sexual, VIH y SIDA en comunidades indígenas de Pando: una aproximación a conocimientos, actitudes y prácticas de poblaciones adultas y jóvenes. Bolivia: FCI/Bolivia; 2007.

19. Programa conjunto de las Naciones Unidas sobre el VIH/Sida, Organización Panamericana de la Salud. El acceso a la atención integral para las personas viviendo con VIH y/o SIDA en Bolivia. La Paz: 2007. 
20. Ministerio de Salud y Deportes. Informe de monitoreo y evaluación de la implementación del compromiso UNGASS sobre el VIH/SIDA en Bolivia. Serie Documentos de Divulgación Científica. La Paz: UNAIDS; 2006.

21. Ministerio de Salud y Deportes. UNGASS Bolivia, 2006-2007. Informe sobre la respuesta Nacional a la Epidemia del VIH/SIDA en Bolivia. La Paz: Ministerio de Salud y Deportes. Unidad Nacional de Epidemiología. Programa Nacional ITS/VIH/SIDA; 2007.

22. Ministerio de Salud y Deportes y Programa Nacional De ITS/VIH/SIDA. Estudio sobre sexualidad masculina y VIH en Bolivia. Disponible en: http://www.sns.gov.bo/its-vih-sida/Publicaciones/Investigaciones/Estudios-TB-vih.pdf

23. Ramírez Hita S. La contribución del método etnográfico en el registro del dato epidemiológico. Epidemiología sociocultural indígena quechua de la ciudad de Potosí. Salud Colectiva (Buenos Aires) 2009; 5(1): 63-85.

Recibido: 27 de julio de 2010

Aceptado: 13 de septiembre de 2010 